\title{
Estridor na sala de parto
}

\author{
Stridor in the delivery room
}

Ana Carolina Rangel ${ }^{1}$, Luciana Figueira Pegorer ${ }^{2}$, Ricardo Vilela ${ }^{3}$

\section{RESUMO}

Objetivo: Ressaltar a importância do estridor por anel vascular em recém-nascidos.

Descrição do caso: Caso 1: recém-nascido a termo do sexo feminino, com história prévia de poli-hidrâmnio, apresentou estridor na sala de parto e necessidade de intubação orotraqueal com parâmetros ventilatórios moderados. Trouxe melhora do quadro respiratório após a inserçào de cânula traqueal além do habitual, o que motivou a investigação de compressão traqueal extrínseca. Realizado diagnóstico de duplo arco aórtico, seguido de cirurgia corretiva com boa evolução. Caso 2: menina de sete meses com estridor desde o nascimento e diagnóstico prévio de laringomalácia, com diversas internações anteriores por doenças respiratórias. Na última internação, houve melhora dos sintomas após a intubação seletiva do brônquio principal direito, sugerindo compressão extrínseca da traqueia. Após a confirmação do diagnóstico de anel vascular, foi submetida à correção cirúrgica, porém evoluiu a óbito.

Comentários: $\mathrm{O}$ anel vascular não é a principal causa de estridor em recém-nascidos, mas deve ser considerado no diagnóstico diferencial. O baixo índice de suspeição dessa alteração leva a atrasos no tratamento e, como consequência, aumenta o risco de traqueomalácia e dismotilidade esofágica mesmo após a correção cirúrgica.

Palavras-chave: recém-nascido; sons respiratórios; estenose traqueal; aorta torácica.

Instituição: Universidade Estadual de Campinas (Unicamp) e Fundação Centro Médico de Campinas, Campinas, SP, Brasil

${ }^{1}$ Médica Residente de Pediatria da Fundação Centro Médico de Campinas, Campinas, SP, Brasil

2Médica Residente de Medicina Intensiva Pediátrica do Hospital de Clínicas da Unicamp, Campinas, SP, Brasil

${ }^{3}$ Mestre em Pediatria pela Faculdade de Ciências Médicas da Unicamp; Médico Assistente da Unidade de Terapia Intensiva Pediátrica da Fundação Centro Médico de Campinas e do Hospital de Clínicas da Unicamp, Campinas, SP, Brasil

\section{ABSTRACT}

Objective: To underline the importance of vascular rings causing stridor in newborns infants.

Case description: Case 1: term female newborn infant with prenatal history of polyhydramnios that presented stridor in the delivery room. She was treated with endotracheal intubation and moderate ventilator settings. She improved her respiratory distress after the insertion of the endotracheal tube deeper than usual. This fact prompted the investigation of extrinsic tracheal compression. A double aortic arch was diagnosed, which had satisfactory surgical outcome. Case 2: a seven-month old girl with stridor since birth and previous diagnosis of laryngomalacia had several hospital admissions due to respiratory distress. In her last hospital stay, she improved after a selective intubation of the right main bronchus, suggesting the presence of extrinsic tracheal compression. After the diagnosis of a vascular ring, she was submitted to the surgical correction, but she died soon after surgery.

Comments: Vascular rings are not the main cause of upper airway obstruction in newborn infants but they should be considered in the differencial diagnosis. The low index of suspicion delays diagnosis and treatment and increases the risk of tracheomalacia and esophageal dysmotility, even after surgical repair.

Key-words: infant, newborn; respiratory sounds; tracheal stenosis; aorta, thoracic.
Endereço para correspondência:

Ana Carolina Rangel

Rua Sylvio Baratelli, 58, Bloco 1

CEP 13073-037 - Campinas/SP,

E-mail: carolrac@unisys.com.br

Conflitos interesses: nada a declarar

Recebido em: 20/10/2009

Aprovado em: 4/3/2010 


\section{Introdução}

Doenças congênitas e adquiridas das vias aéreas podem causar estridor, dispneia, tosse, sibilos, disfagia e infecções recorrentes do trato respiratório. A maioria desses sintomas é comum na infância, mas sua persistência deve ser um sinal de alerta ao pediatra. A causa mais frequente de estridor em crianças à avaliação endoscópica é a laringomalácia em $41 \%$ dos casos, seguida pela estenose subglótica em $18 \%$, sendo rara a compressão vascular extrínseca da traqueia $(2 \%)^{(1)}$. Considerando-se apenas o primeiro ano de vida, a laringomalácia e a compressão traqueal vascular extrínseca são as causas mais comuns de estridor ${ }^{(2)}$.

Entre os diagnósticos diferenciais de estridor em recémnascidos estão: estenose subglótica congênita, paralisia de cordas vocais, cisto de valécula, duplicação traqueal, aneurisma de ducto arterioso, cisto ou hemangioma subglótico e agenesia traqueal e laríngea ${ }^{(2-4)}$.

O objetivo deste relato de casos foi ressaltar a importância do diagnóstico de anel vascular o mais precocemente possível, principalmente quando os sinais e sintomas se manifestam já no período neonatal. O desconhecimento dessa anomalia e, em consequencia, o baixo índice de suspeita são fatores importantes para o atraso no tratamento, aumentando o risco de traqueomalácia, dismotilidade esofágica e persistência dos sintomas mesmo após a correção cirúrgica ${ }^{(5)}$.

O Comitê de Ética em Pesquisa da Faculdade de Ciências Médicas da Universidade Estadual de Campinas (Unicamp) aprovou este artigo para publicação sem restrições.

\section{Descrição de casos}

\section{Caso 1}

Recém-nascido do sexo feminino, com antecedente de polihidrâmnio e sem alterações morfológicas ao ultrassom. Parto cesáreo, peso ao nascimento de $4230 \mathrm{~g}$, idade gestacional de 38 semanas e Apgar de 7 e 8 no $1^{\circ}$ e $5^{\circ}$ minutos, respectivamente. Nasceu com boa vitalidade e chorando, com estridor e grande quantidade de secreção fluida em via aérea alta. Mantinha cianose no primeiro minuto, com esforço respiratório inefetivo. Houve melhora com ventilação com pressão positiva e uso de oxigênio, porém evoluiu com bradicardia. Realizou-se a intubação orotraqueal, após a qual manteve taquipneia com roncos inspiratórios à ausculta pulmonar e hepatomegalia. Recebeu ventilação mecânica com parâmetros moderados. A readiografia de tórax mostrou hiperinsuflação, discreta opacidade heterogênea peri-hilar e em ápice direito e tubo orotraqueal $2 \mathrm{~cm}$ acima da carina (Figura 1 ).
A ausculta pulmonar passou a apresentar tempo expiratório prolongado e sibilos inspiratórios e expiratórios. A paciente mostrou piora do padrão respiratório obstrutivo, independentemente das pressões ventilatórias, sugerindo estenose fixa e não apenas um colapso dinâmico do tipo malácia. A cânula orotraqueal foi introduzida a $11 \mathrm{~cm}$ do lábio superior, sem intubação seletiva, com melhora considerável da ausculta pulmonar e desaparecimento do estridor, o que permitiu diminuir o suporte ventilatório.

Realizou-se então tomografia computadorizada de tórax sugestiva de anel vascular. Posteriormente, a angiorressonância comprovou a presença de duplo arco aórtico envolvendo o segmento distal da traqueia e o esôfago médio, com importante estreitamento de ambas as estruturas.

No sétimo dia de internação, realizou-se cirurgia com toracotomia esquerda para secção do arco posterior e ligadura de canal arterial, seguida de extubação no sétimo dia de pós-operatório e alta hospitalar no $14^{\circ}$ dia. No seguimento, após dois anos, apresentava-se eupneica, apenas com tosse muito ruidosa nos episódios ocasionais de infecções de vias aéreas superiores.

\section{Caso 2}

Menina de sete meses, com antecedente de estridor desde o nascimento. A suspeita clínica inicial foi de laringomalácia; no entanto, a nasofibroscopia evidenciou provável membrana na comissura laríngea anterior. A criança teve três internações prévias por insuficiência respiratória, com diagnóstico clínico de pneumonia, bronquiolite e laringotraqueomalácia.

Foi trazida ao pronto-socorro com piora da tosse e da dispneia há um dia. Apresentava desconforto respiratório importante, caracterizado por estridor e retração da fúrcula esternal. Após inalação com adrenalina e administração de dexametasona intramuscular, passou-se a observar gemência, sibilância e tempo expiratório prolongado. Realizaram-se inalações com fenoterol, sem mudança do quadro clínico, evoluindo com piora que motivou a transferência para a Unidade de Terapia Intensiva Pediátrica (UTIP). A criança foi intubada, sendo iniciada a ventilação mecânica, porém mantinha o quadro de desconforto obstrutivo baixo.

Em episódio de piora abrupta, optou-se pela troca da cânula traqueal. Houve dificuldade de progressão subglótica e, depois de vencida a obstrução, houve melhora significativa do desconforto. O controle radiológico mostrava intubação seletiva do brônquio fonte direito, sugerindo obstrução traqueal baixa. 
A broncoscopia evidenciou obstrução da luz traqueal em terço distal, correspondendo a uma obstrução extrínseca e pulsátil. A tomografia de tórax sugeria duplicidade do arco aórtico. Para confirmação e planejamento cirúrgico, realizouse angiotomografia, que confirmou o duplo arco aórtico e a compressão do terço distal da traqueia (Figura 2).

No pós-operatório, permaneceu com intubação orotraqueal, voltou a apresentar sibilância com a fixação adequada da cânula orotraqueal para a idade (a $10 \mathrm{~cm}$ do lábio superior), que melhorou com a introdução até $14 \mathrm{~cm}$. Foi repetida a broncoscopia, que mostrou permanência do estreitamento traqueal dinâmico sugestivo de traqueomalácia. Dois dias após, a paciente faleceu por asfixia devida a sangramento traqueal maciço.

\section{Discussão}

O sistema de arcos branquiais dá origem a seis pares de arcos aórticos primitivos que, na vida fetal, originam parte do arco aórtico e da artéria pulmonar. Os anéis vasculares são decorrentes de uma regressão anormal do quarto e quinto arcos do sistema branquial, resultando em compressão da traqueia e do esôfago ${ }^{(6)}$. São malformações raras e se subdividem em anéis completos (duplo arco aórtico e arco aórtico à direita com persistência do ducto arterioso) e incompletos (artéria subclávia direita anômala e anel de artéria pulmonar) ${ }^{(5)}$.

O anel vascular frequentemente é assintomático ao nascimento e suas manifestações podem surgir após meses ou anos. Os sinais e sintomas mais comuns são dispneia, estridor, tosse, sibilos e disfagia ${ }^{(7)}$. Uma forte suspeição deve ocorrer quando há presença de sintomas respiratórios recorrentes e que não respondem adequadamente ao tratamento convencional, por exemplo, ao uso de broncodilatadores, como no segundo caso apresentado. Quando presentes em neonatos, as evidências variam desde estridor, sibilos e infecções respiratórias de repetição até obstruções agudas e graves, acompanhadas de apneia e episódios de cianose, que podem acompanhar-se de bradicardia ${ }^{(2,8)}$.

A avaliação do recém-nascido com estridor inicia-se pela laringoscopia que, em geral, leva ao diagnóstico de laringomalácia ${ }^{(9)}$. No entanto, existe a possibilidade da coexistência de mais de um fator causal de estridor, conforme observado no segundo caso.

$\mathrm{Na}$ investigação do anel vascular, o primeiro exame a ser realizado é a radiografia de tórax, que normalmente mostra graus variados de atelectasia e hiperinsuflação ${ }^{(7)}$, como descrito no primeiro paciente e mostrado na Figura 1. A técnica recomendada utiliza alta quilovoltagem na

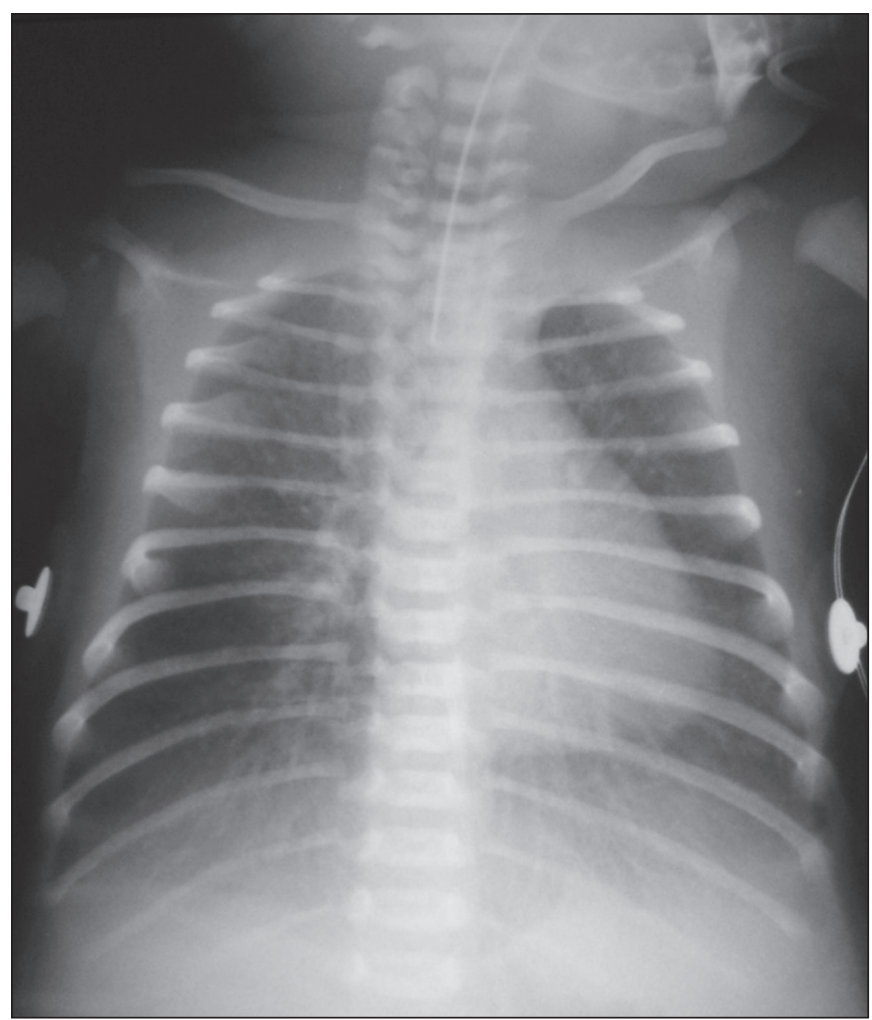

Figura 1 - Radiografia do primeiro caso mostra hiperinsuflação pulmonar. A extremidade distal da cânula traqueal está no terço médio da traqueia, acima do anel vascular.

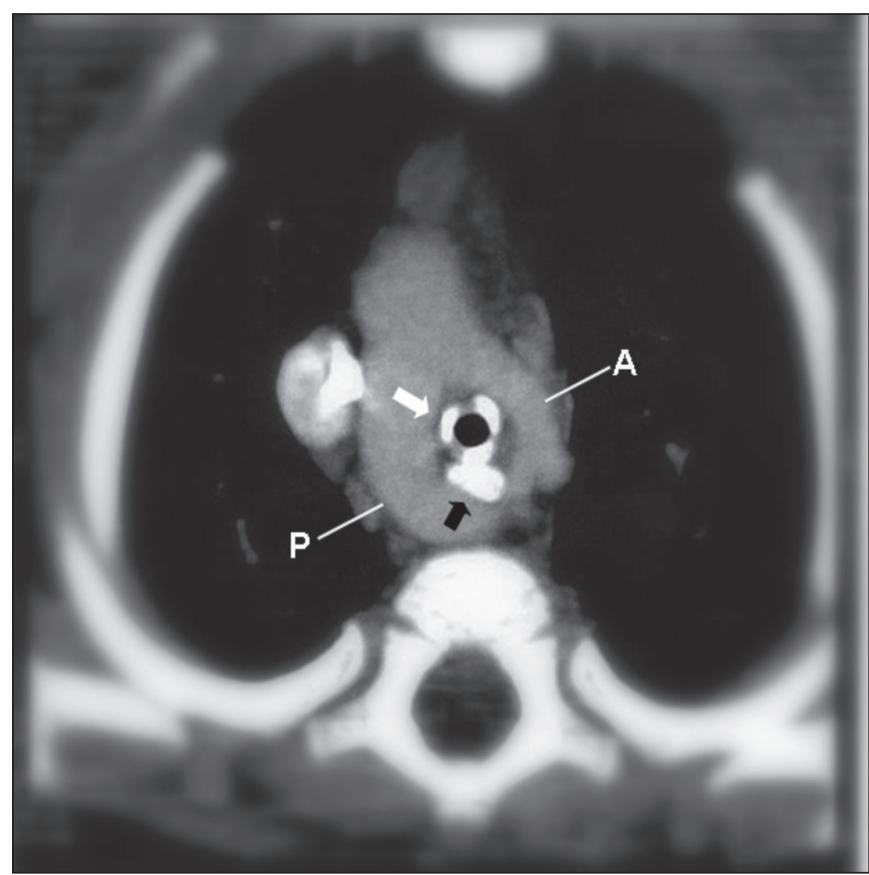

Figura 2 - Angiorressonância torácica do segundo caso. Observam-se o ramo anterior $(A)$ e o ramo posterior $(P)$ do duplo arco aórtico envolvendo a cânula traqueal (seta branca), além da sonda orogástrica (seta preta). 
incidência anteroposterior e lateral para melhor visualização da via aérea ${ }^{(10)}$.

O esofagograma não-invasivo e de realização simples, associado ao exame endoscópico, é o principal método diagnóstico ${ }^{(5)}$. Alguns estudos relatam precisão diagnóstica em $80^{(11)}$ a $90 \%^{(5)}$ dos casos. O esofagograma mostra compressão posterior do esôfago em todos os portadores de anomalias vasculares, exceto no anel de artéria pulmonar ${ }^{(6,7)}$. Porém, é importante ressaltar que a anatomia exata do anel vascular não pode ser determinada por esse exame ${ }^{(6)}$. A broncoscopia, por outro lado, é um exame invasivo e que pode levar a uma piora do quadro clínico. No entanto, a broncoscopia é útil em pacientes impossibilitados de realizarem o esofagograma ${ }^{(2,5)}$. O exame considerado referência é a arteriografia, mas também trata-se de procedimento invasivo e de risco ${ }^{(5,6)}$, podendo não ser eficiente se o vaso a ser estudado for atrésico ${ }^{(4)}$. O ideal é escolher o método de imagem de acordo com as condições clínicas do paciente, a disponibilidade do serviço em que se encontra e a aptidão do examinador. Dentre as outras possibilidades diagnósticas, o ecocardiograma não é capaz de avaliar o tipo de anel vascular e se ele está presente ou não ${ }^{(12)}$. Sua realização, contudo, é recomendada posteriormente ao diagnóstico, uma vez que anomalias cardíacas estão associadas em $12^{(11)}$ a $29 \%^{(6)}$ dos casos.

Em algumas instituições ${ }^{(5,9,12)}$, a radiografia de tórax e o esofagograma são considerados suficientes para indicar a cirurgia de correção. Em outros locais ${ }^{(7,10,13)}$, entretanto, realiza-se a tomografia computadorizada ou a ressonância

\section{Referências bibliográficas}

1. Lubianca-Neto JF, Fischer GB, Peduzzi FD, L-Junior H, Krumenauer RC, Richter VT. Achados clínicos e endoscópicos em criança com estridor. Rev Bras Otorrinolaringol 2002;68:314-8.

2. Park JG, Wylam ME. Congenital stridor: unusual manifestation of coarctation of the aorta. Pediatr Cardiol 2006;27:137-9.

3. Kaushal M, Upadhyay A, Aggarwal R, Deorari AK. Congenital stridor due to bilateral vocal cord palsy. Indian J Pediatr 2005;72:443-4.

4. Hsieh WS, Yang PH, Wong KS, Li HY, Wang EC, Yeh TF. Vallecular cyst: an uncommon cause of stridor in newborn infants. Eur J Pediatr 2000;159:79-81.

5. Longo-Santos LR, Maksoud-Filho JG, Tannuri U, Andrade WC, Gonçalves $\mathrm{ME}$, Cardoso SR et al. Vascular rings in childhood: diagnosis and treatment. J Pediatr (Rio J) 2002;78:244-5.

6. Humphrey C, Duncan K, Fletcher S. Decade of experience with vascular rings at a single institution. Pediatrics 2006;117:e903-8.

7. Backer CL, Mavroudis C, Rigsby CK, Holinger LD. Trends in vascular ring surgery. J Thorac Cardiovasc Surg 2005;129:1339-47.

8. Abdel-Rahman U, Ahrens P, Fieguth HG, Kitz R, Heller K, Moritz A. Surgical treatment of tracheomalacia by bronchoscopic monitored aortopexy in infants and children. Ann Thorac Surg 2002;74:315-9.

9. Alvarez CG, Holmgren LP, Caussade SL, Paz FC, Jofré DP, Sánchez ID. Estridor de causa inhabitual en lactantes. Descripción de 3 casos. Rev Chil nuclear magnética com o intuito de planejar o ato operatório com maior precisão. Esses são exames importantes quando há necessidade de afastar outras causas de compressão extrínseca da traqueia, como cistos e tumores mediastinais ${ }^{(5)}$.

A cirurgia precoce deve ser realizada, pois o reparo tardio está associado à menor reversibilidade de traqueobroncomalácia ${ }^{(6)}$. Nos casos em que o diagnóstico é feito tardiamente, as complicações clínicas mais frequentes são traqueomalácia, dismotilidade esofágica e sibilância recorrente ${ }^{(5)}$, conforme visto no segundo caso.

Normalmente, os sintomas e sinais de compressão traqueal e do esôfago melhoram logo após a cirurgia de correção, porém não desaparecem completamente antes de seis meses ${ }^{(9,14,15)}$. A regressão lenta dos sintomas respiratórios é esperada, devido à pneumopatia pré-existente ou à traqueomálacia remanescente. Sintomas respiratórios ou gastrintestinais residuais ocorrem em $13 \%$ das crianças e a mortalidade no pós-operatório imediato é de 3,3\%(16). A cirurgia de correção do anel vascular associado à compressão traqueal tem uma mortalidade maior do que a correção do anel sem sintomas respiratórios obstrutivos ${ }^{(13)}$. A mortalidade tardia está relacionada à pneumopatia crônica, infecções de repetição e traqueomalácia grave ${ }^{(7)}$.

É importante ressaltar que existem numerosos diagnósticos para o estridor em recém-nascidos. $\mathrm{O}$ anel vascular não é o primeiro diagnóstico a ser avaliado nesses casos por ser menos comum, porém deve ser considerado quando a investigação inicial ou a terapêutica são mal sucedidas.
Pediatr 2002;73:152-8.

10. Berdon WE. Rings, slings, and other things: vascular compression of the infant trachea updated from the midcentury to the millennium - the legacy of Robert E. Gross, MD, and Edward B. D. Neuhauser, MD. Radiology 2000;216:624-32.

11. Bonnard A, Auber F, Fourcade L, Marchac V, Emond S, Révillon Y. Vascular ring abnormalities: a retrospective study of 62 cases. J Pediatr Surg 2003; 38:539-43.

12. Riedi CA, Rosário Filho NA, Trevisan IV, Carreiro JE, Escussiato DL. Malformações pulmonares e mediastinais com repercussões respiratórias. $J$ Pneumol 1998;24:303-10.

13. Chiu PP, Rusan M, Williams WG, Caldarone CA, Kim PC. Long-term outcomes of clinically significant vascular rings associated with congenital tracheal stenosis. J Pediatr Surg 2006;41:335-41.

14. Colquhoun IW, Pollock JC. Distal tracheal stenosis in two children with double aortic arch and associated tracheomalacia. Eur J Cardiothorac Surg 1990;4:287-9.

15. Yilmaz M, Ozkan M, Dogan R, Demircin M, Ersoy U, Boke E et al. Vascular anomalies causing tracheoesophageal compression: a 20-year experience in diagnosis and management. Heart Surg Forum 2003;6:149-52.

16. Martins RG, Dias NH, Castilho EC, Trindade SH. Endoscopic findings in children with stridor. Rev Bras Otorrinolaringol 2006;72:649-53. 